\title{
Propagação vegetativa de Brosimum gaudichaudii Tréc. (mama-cadela) por estacas de raízes
}

\author{
SILVA, D.B. ${ }^{1 *}$; VIEIRA, R.F.'; CORDEIRO, M.C.T.';PEREIRA, E.B.C. ${ }^{2}$; PEREIRA, A.V. ${ }^{3}$ \\ ${ }^{1}$ Embrapa Recursos Genéticos e Biotecnologia, Caixa Postal 02372, CEP: 70770-900, Brasília-Brasil \\ *dijalma@cenargen.embrapa.br 22Agencia Rural, Caixa Postal 331, Setor Leste Universitário, CEP: 74.610-060, \\ Goiânia-Brasil ${ }^{3}$ Embrapa Transferência de Tecnologia, Escritório de Negócios de Goiânia, Caixa Postal 714, \\ CEP: 74.001-970, Goiânia-Brasil
}

\begin{abstract}
RESUMO: Brosimum gaudichaudiiTréc. (mama-cadela) é uma planta medicinal nativa do Cerrado, utilizada na medicina tradicional. $O$ objetivo deste trabalho foi verificar a possibilidade de propagação de mama-cadela por meio de estacas de raízes, sob o efeito de reguladores de crescimento e de diferentes substratos. No experimento 1 foram avaliados os efeitos da aplicação de ácido indolbutírico (AIB) e ácido naftaleno-acético (ANA) na concentração de $1000 \mathrm{mg} \mathrm{L}^{-1}$ e três substratos (S1- areia; S2 - $75 \%$ de areia $+25 \%$ de substrato comercial; S3 $-50 \%$ de areia $+50 \%$ de substrato comercial). No experimento 2 foram avaliadas 4 doses de AIB: 0 - testemunha; $250 \mathrm{mg}$ $\mathrm{L}^{-1}(1,3426 \mathrm{mM}) ; 500 \mathrm{mg} \mathrm{L}^{-1}(2,6853 \mathrm{mM})$ e $1000 \mathrm{mg} \mathrm{L}^{-1}(5,3706 \mathrm{mM})$; e 4 doses de ácido naftaleno-acético (ANA): 0 - testemunha; $250 \mathrm{mg} \mathrm{L}^{-1}(1,2295 \mathrm{mM}) ; 500 \mathrm{mg} \mathrm{L}^{-1}(2,458 \mathrm{mM})$; e 1000 $\mathrm{mg} \mathrm{L}^{-1}(4,918 \mathrm{mM})$. O delineamento experimental utilizado foi de blocos ao acaso com três repetições de seis estacas por parcela. No experimento 1, os substratos compostos por areia e areia $(75 \%)$ + substrato comercial (25\%) proporcionaram os maiores Índices de pegamento. A aplicação de AIB (1000 $\mathrm{mg} \mathrm{L}^{-1}$ ) proporcionou aumentos relativos de 30,8\% e 51,3\%, no IMP quando comparada com a testemunha e a aplicação de ANA, respectivamente. No experimento 2, observou-se resposta quadrática significativa da aplicação de AIB sobre o IMP. A dose de 500 $\mathrm{mg} \mathrm{L}^{-1}(2,6853 \mathrm{mM})$ promoveu maior IMP. Não houve efeito significativo das doses de ANA para os parâmetros avaliados. Não houve efeito significativo relevante de substratos ou hormônios sobre os demais parâmetros avaliados. Estes resultados evidenciam o potencial de utilização de estacas de raiz de mama-cadela para a produção de mudas clonais desta espécie.
\end{abstract}

Palavras-chave: plantas medicinais, cerrado, reguladores de crescimento, substrato

ABSTRACT: Vegetative propagation of Brosimum gaudichaudii Tréc. (mama-cadela) by root cuttings. Brosimum gaudichaudii Tréc. (mama-cadela) is a medicinal plant native to Cerrado and largely used in traditional medicine. The aim of this study was to verify the propagation of mama-cadela by means of root cutting under the effect of plant growth regulators and different substrates. In experiment 1, the effects of indole butyric acid (IBA) and naphthylacetic acid (NAA) at the concentration of $1000 \mathrm{mg} \mathrm{L}^{-1}$ were evaluated together with three substrates (S1 - sand; S2 - $75 \%$ sand $+25 \%$ commercial substrate; S3 - 50\% sand + 50\% commercial substrate). In experiment 2, 4 IBA levels were evaluated: 0 - control; $250 \mathrm{mg} \mathrm{L}^{-1}(1.3426 \mathrm{mM}) ; 500 \mathrm{mg} \mathrm{L}^{-1}$ (2.6853 mM) and $1000 \mathrm{mg} \mathrm{L}^{-1}(5.3706 \mathrm{mM})$, as well as 4 NAA levels: 0 - control; $250 \mathrm{mg} \mathrm{L}^{-1}$ (1.2295 mM); $500 \mathrm{mg} \mathrm{L}^{-1}(2.458 \mathrm{mM})$ and $1000 \mathrm{mg} \mathrm{L}^{-1}(4.918 \mathrm{mM})$. The adopted experimental design was in randomized blocks with three replicates of six cuttings per plot. In experiment 1 , substrates containing sand and sand $(75 \%)+$ commercial substrate $(25 \%)$ promoted the highest average rooting indexes. Application of IBA $\left(1000 \mathrm{mg} \mathrm{L}^{-1}\right)$ led to increases of $30.8 \%$ and $51.3 \%$ in the average rooting indexes compared to control and NAA application, respectively. In experiment 2 , there was a significant quadratic response of IBA application on the average rooting index. The level of $500 \mathrm{mg} \mathrm{L}^{-1}(2.6853 \mathrm{mM})$ promoted the highest average rooting index. There was no significant effect of NAA levels for the evaluated parameters. Similarly, there was no significant effect of substrates or hormones on the remaining parameters evaluated. These results show the potential use of mama-cadela root cuttings for the production of clonal seedlings of this species.

Key words: medicinal plants, Cerrado, growth regulators, substrate

Recebido para publicação em 03/04/2009

Aceito para publicação em 26/01/2010

Rev. Bras. Pl. Med., Botucatu, v.13, n.2, p.151-156, 2011. 


\section{INTRODUÇÃO}

Brosimum gaudichaudii Tréc. (Moraceae) é uma planta nativa do Brasil, conhecida popularmente como mama-cadela e considerada prioritária para a conservação e manejo de recursos genéticos vegetais (Vieira et al., 2002). Esta espécie, encontrada tanto em áreas de cerrado como da floresta amazônica e mata atlântica, é o único representante do gênero Brosimum na vegetação do cerrado (Palhares et al., 2006). Os frutos carnosos são comestíveis e a madeira é utilizada em marcenaria. As folhas, casca e as raízes são usadas como medicamento pelas populações da região do Brasil Central principalmente, para o tratamento do vitiligo (Lorenzi, 1988; Almeida et al., 1998).

De acordo com Pozetti (2005), esta espécie foi bastante estudada sob os aspectos químicos, farmacológicos e toxicólogicos, permitindo comprovar sua atividade fotossensibilizante, preconizada pelo uso popular. O vitiligo, de ocorrência maior em habitantes de regiões tropicais, é uma afecção cutânea adquirida, de etiologia desconhecida, caracterizada pela ausência de pigmentação da pele (hipomelanose) circundada por área de pigmentação normal (Bennet \& Plum, 1996; Thomas, 2000). Os principais constituintes ativos da planta são as furanocumarinas; bergapteno e psoraleno de propriedades fotossensibilizantes, produzidas pelas rotas de metabolismo secundário (Pozeti \& Bernardi, 1969). Além do uso tradicional da mama-cadela, derivados psoralênicos são encontrados em medicamentos como o Viticromin $\AA$, elaborado a partir de cascas da porção inferior do caule e das raízes de mamacadela, que depois de colhidas, são dessecadas, descascadas, trituradas e preparadas em formas farmacêuticas para uso interno (comprimidos) e externo (pomada e loção) (Anatel et al., 2005). De acordo com Bucher (2002), a demanda por esta espécie, sem reposição, tem diminuído as populações naturais da planta, e por falta de investimentos em técnicas de propagação ou pesquisas sobre reprodução, vem sendo dizimada pelo extrativismo predatório.

Palhares et al. (2006) observaram que a planta apresenta rápido crescimento radicular e presença de xilopódio que lhe confere vantagens competitivas para o estabelecimento e sobrevivência no cerrado, diante das adversidades naturais como a seca anual e às queimadas, naturalmente recorrentes neste ambiente. Além da capacidade de rebrotamento do xilopódio, plantas adultas desta espécie apresentam raízes gemíferas longas, plagiotrópicas originando ramos caulinares aéreos, podendo constituir populações clonais (Palhares et al., 2006; Jacomassi et al., 2007). A rebrota de espécies arbóreas a partir de raízes gemíferas tem sido considerada um dos principais mecanismos de regeneração natural em ambientes perturbados (Rizzini \& Heringer, 1966, Penha, 2004), inclusive em áreas mineradas, onde Corrêa et al. (2007) observaram que dentre as 98 espécies lenhosas encontradas nas áreas mineradas do Distrito Federal, Brosimum gaudichaudii foi a quarta espécie de maior dominância do estrato lenhoso, apresentando índice de 8,59\% de dominância.

Brosimum gaudichaudii apresenta sementes com alta germinabilidade, tanto em condições de laboratório como em condições de viveiro, (Machado et al., 1986; Machado \& Parente, 1986; Silva et al., 2001; Sales et al., 2002). Mas, estudos sobre fisiologia de sementes realizados por Wetzel (1997) indicaram que as sementes desta espécie, embora com alta capacidade germinativa, apresentam comportamento fisiológico do tipo recalcitrante. O termo recalcitrante é atribuído às sementes que apresentam altos teores de umidade no estádio de maturação fisiológica, não toleram a secagem e não podem ser armazenadas em temperaturas abaixo de zero, em longo prazo (Roberts, 1973; Ellis et al., 1990; Ellis et al., 1991; Silva et al., 2007). Sementes desta espécie que foram desidratadas por Wetzel (1997) ainda apresentavam teores de umidade acima de $10 \%$, e nenhuma porcentagem de germinação. Palhares et al. (2006), tomando como base, os trabalhos de Martins (1998), Fidelis (1998) e Bucher (2002), comentam que as tentativas de propagação in vitro da espécie não foram, ainda, bem-sucedidas. Desta forma, é imprescindível a adoção de novas estratégias para a propagação de Brosimum gaudichaudii.

A propagação vegetativa tem sido praticada com sucesso para várias espécies comerciais. Estacas oriundas das partes aéreas da planta têm sido bastante utilizadas para a propagação, enquanto as estacas de raízes têm sido pouco usadas (Hoffmann et al., 2005). A capacidade de enraizamento das estacas depende principalmente, da família, espécie, idade da planta, época do ano e da parte da planta a ser utilizada (Hartmann et al., 2002 ; Fachinello et al., 2005).

Segundo Melo et al. (1998), a propagação vegetativa de espécies do cerrado ainda é pouco estudada. Até o momento, não foram relatados estudos sobre a propagação vegetativa de Brosimum gaudichaudii através de estacas.

Considerando a grande capacidade natural de rebrota das plantas de mama-cadela em áreas queimadas ou perturbadas, é possível que estacas de raízes desta espécie possam ser usadas para a propagação.

Uma vez definido o tipo de estaca ideal, a escolha do substrato e de técnicas de estímulo ao enraizamento é de fundamental importância para viabilizar a propagação vegetativa. Segundo Hoffmann et al. (2005), o substrato deve proporcionar adequado equilíbrio de umidade, aeração, consistência, nutrientes,

Rev. Bras. PI. Med., Botucatu, v.13, n.2, p.151-156, 2011. 
ausência de patógenos e sementes de plantas infestantes para o bom desenvolvimento da muda. Existem vários tipos de substratos disponíveis no mercado os quais devem ser ajustados para as necessidades da espécie e da parte da planta a ser propagada.

A capacidade da estaca emitir raízes depende de fatores endógenos como balanço hormonal e reserva nutricional da planta. Em muitas espécies, o enraizamento pode ser estimulado por substâncias exógenas como os reguladores de crescimento. A utilização destas substâncias no enraizamento de estacas para a produção de mudas é uma prática bastante difundida em muitas espécies (Fachinello et al., 1995). Os reguladores de crescimento mais usados para o enraizamento de estacas tem sido o ácido naftalenacético (ANA) e o ácido indolbutírico (AIB) em diferentes formulações, concentrações e formas de aplicação (Hinojosa, 2005).

O objetivo deste trabalho foi verificar a possibilidade de propagação de Brosimum gaudichaudii por meio de estacas de raízes, sob o efeito de reguladores de crescimento e de diferentes substratos.

\section{MATERIAL E MÉTODO}

Os experimentos foram conduzidos em telado na Embrapa Recursos Genéticos e Biotecnologia, Brasília, DF. no período de junho de 2003 à julho de 2004. O delineamento experimental utilizado foi de blocos ao acaso com três repetições de seis estacas por parcela. As estacas de raízes com sete centímetros de comprimento, oriundas de plantas jovens ( 2 anos) foram plantadas na posição vertical, em tubetes com $5 \mathrm{~cm}$ de diâmetro por $19 \mathrm{~cm}$ de comprimento, distribuídos em bandejas, sobre bancadas, deixando aproximadamente $1,5 \mathrm{~cm}$ de superfície exposta (sem cobertura de substrato). As irrigações foram realizadas a cada dois dias.

No experimento 1, foram avaliados os efeitos da aplicação e não aplicação (testemunha) de ácido indol-butírico e ácido naftaleno-acético (ANA) na concentração de $1000 \mathrm{mg} \mathrm{L}^{-1}$ correspondendo a 5,3706 $\mathrm{mM}$ (milimolar) de AIB e 4,918 mM (milimolar) de (ANA), respectivamente e três substratos (S1- areia; S2 - $75 \%$ de areia $+25 \%$ de substrato comercial; S3 - $50 \%$ de areia $+50 \%$ de substrato comercial).

No experimento 2 foram avaliadas quatro doses de ácido indol-butírico (AIB): 0 - testemunha; $1,3426 \mathrm{mM}$ (250 mg L-1);2,6853 mM (500 $\left.\mathrm{mg} \mathrm{L}^{-1}\right)$ e 5,3706 mM (1000 $\mathrm{mg} \mathrm{L}^{-1}$ ); e quatro doses de ácido naftaleno-acético (ANA): 0 - testemunha; $1,2295 \mathrm{mM}^{2}\left(250 \mathrm{mg} \mathrm{L}^{-1}\right) ; 2,458 \mathrm{mM}$ (500 $\left.\mathrm{mg} \mathrm{L}^{-1}\right)$; e 4,918 mM (1000 $\left.\mathrm{mg} \mathrm{L}^{-1}\right)$.

Foram avaliados a altura da maior brotação (AMB); número médio de folhas (NF), número médio de brotações (NB) e o Índice médio de pegamento das estacas (IMP). Para o cálculo do IMP, elaborouse uma escala de referência utilizando os seguintes parâmetros: $A=$ no de estacas sem brotações caulinares e sem raiz; $B=n \circ$ de estacas com brotações caulinares e sem raiz; $C=n$ ㅇ de estacas com brotações caulinares e com raiz; $D=n \circ$ de estacas com brotações caulinares e com raíz pivotante. Atribuiu-se os pesos 0, 1, 2 e 3 aos parâmetros avaliados em relação ao pegamento das estacas, respectivamente. Em seguida, calculouse o IMP através da fórmula:

IMP $=[(A \times 0)+(B \times 1)+(C \times 2)+(D \times 3)] /($ № de estacas por parcela $=6$ )

O substrato comercial usado foi o Plantimax ${ }^{R}$. Cada substrato foi fertilizado com osmocote $\left(3 \mathrm{~g} \mathrm{~L}^{-1}\right)$. Osmocote é um fertilizante de liberação lenta contendo $\mathrm{N}(15 \%), \mathrm{P}_{2} \mathrm{O}_{5}(10 \%), \mathrm{K}_{2} \mathrm{O}$ $(10 \%), \mathrm{Ca}(3,5 \%), \mathrm{Mg}(1,5 \%), \mathrm{S}(3 \%), \mathrm{B}(0,02 \%), \mathrm{Cu}$ $(0,05 \%)$, Fe $(0,5 \%)$, Mn $(0,1 \%)$, Zn $(0,05 \%)$ e Mo $(0,004 \%)$. As estacas foram distribuídas em blocos em função do diâmetro médio: $0,86 \mathrm{~cm}$ (Bloco 1); $0,67 \mathrm{~cm}$ (Bloco 2); 0,35 cm (Bloco 3).

O substrato utilizado no experimento 2 foi composto por $75 \%$ areia $+25 \%$ substrato comercial e adubado com osmocote $\left(3 \mathrm{~g} \mathrm{~L}^{-1}\right)$. O diâmetro médio das estacas foi de $0,67 \mathrm{~cm}$.

Em ambos experimentos a fonte de AIB foi 0 sal de potássio puro e a de ANA o produto comercial Raizon $^{R}$. Os tratamentos com os reguladores de crescimento foram realizados por imersão da parte basal das estacas em solução de cada produto, pelo período de cinco segundos, antes do plantio. Os resultados foram analisados pelo programa SAS e as médias comparadas pelo Teste de Tukey a $5 \%$ de probabilidade.

\section{RESULTADO E DISCUSSÃO}

\section{Experimento 1}

Não houve efeito significativo da interação entre substratos e aplicação de reguladores de crescimento para nenhum dos parâmetros avaliados. O IMP foi significativamente modificado em função da aplicação de reguladores de crescimento e dos tipos de substrato (Tabela 1). O substrato S1 (areia) foi significativamente superior ao S3 $(50 \%$ de areia + $50 \%$ de substrato comercial) não diferindo do S2 (75\% de areia $+25 \%$ de substrato comercial). Porem, o substrato S2 favoreceu uma boa agregação física, preservando a integridade das raízes. De acordo com Hoffmann et al. (2005), esta característica é altamente desejável em um substrato. A aplicação de AIB proporcionou aumento significativo de 51,30\%, no IMP, quando comparada com a aplicação de ANA, mas não diferiu significativamente da testemunha. Desta forma, o custo benefício da utilização de AIB para o enraizamento de estacas de mama-cadela deve ser considerado. Também, o tempo de imersão ( $5^{\prime}$ ) pode 
não ter sido suficiente para que o produto fosse absorvido em quantidade suficiente para promover 0 maior estímulo ao enraizamento das estacas.

O número de folhas apresentou efeito significativo dos reguladores de crescimento não se observando o mesmo em resposta aos tipos de

TABELA 1. Efeitos de substratos e hormônios sobre a altura da maior brotação (AMB em $\mathrm{cm}$ ), número médio de folhas das brotações (NF), número médio de brotos (NB) e Índice médio de pegamento (IMP).

\begin{tabular}{lllll}
\hline Tratamentos & \multicolumn{4}{c}{ Parâmetros } \\
\cline { 2 - 5 } & AMB & NF & NB & IMP \\
\hline Substratos** $^{*}$ & & & & \\
\hline S1 & $2,68 a$ & $2,78 a$ & $1,31 a$ & $1,72 a$ \\
S2 & $2,41 a$ & $2,38 a$ & $1,00 a$ & $1,40 a b$ \\
S3 & $1,79 a$ & $2,01 a$ & $1,23 a$ & 1,09 b \\
\hline Reguladores de crescimento (RC) \\
\hline Sem RC & $2,37 a$ & $2,54 a$ & $1,16 a$ & $1,33 a b$ \\
AlB & $2,74 a$ & $3,04 a$ & $1,36 a$ & $1,74 a$ \\
ANA & $1,78 a$ & 1,59 b & $1,03 a$ & $1,15 \quad b$ \\
\hline C. V. (\%) & 78,48 & 68,28 & 54,32 & 31,39 \\
\hline
\end{tabular}

*As médias seguidas da mesma letra no sentido vertical não diferem significativamente entre si pelo teste de Tukey a $5 \%$ de probabilidade. ${ }^{* *} \mathrm{~S} 1$ - areia; S2 $-75 \%$ de areia $+25 \%$ de substrato comercial; S3 $-50 \%$ de areia $+50 \%$ de substrato comercial

substrato. A aplicação de ANA proporcionou redução significativa no número médio de folhas. Os demais parâmetros não foram afetados pelos tratamentos. As estacas com diâmetro médio de 0,87 cm e 0,67 $\mathrm{cm}$ proporcionaram maiores valores para todos os parâmetros. Observou-se que o comprimento das raízes pivotantes variou de $3,5 \mathrm{~cm}$ até $14 \mathrm{~cm}$, sendo maiores nos substratos $\mathrm{S} 1$ e $\mathrm{S} 2$ e no tratamento com AIB (Figura 1).
De um modo geral as médias de todos os parâmetros apresentam uma ordenação nos substratos que decresce no sentido $S 1$, S2 e S3 e nos reguladores de crescimento, no sentido AIB, sem hormônio e ANA. Estas tendências não foram detectadas pela analise estatística devido aos elevados valores observados para os coeficientes de variação. Possivelmente isto se deve ao tamanho das parcelas usadas para experimentação com uma planta não domesticada e, portanto, presumivelmente com elevada variabilidade genética.

\section{Experimento 2}

A Figura 2 apresenta as médias do Índice médio de pegamento (IMP) para as diferentes doses de AIB com seus respectivos intervalos de confiança $(0,95)$. O efeito de doses crescentes de AIB sobre o IMP apresentou componente quadrático significativo $F_{(1,15)}=3,79(p=0,070)$. A dose de 2,6853 mM (500 $\left.\mathrm{mg}^{-1}\right)$, proporcionou o maior Índice médio de pegamento. Não houve efeito significativo das doses de ANA para nenhum parâmetro avaliado (Tabela 2) e nem da aplicação de AIB sobre altura das brotações, número total de folhas e número total de brotos das estacas (Tabela 3).

Estes resultados podem ser considerados bastante satisfatórios, quando comparados com os obtidos por Pio et al. (2008) com estacas de raízes de figueira, espécie domesticada da mesma família da mama-cadela, as quais apresentaram um bom enraizamento com a aplicação de AIB (2000 mg L-1), porém com ausência de brotações, inviabilizando a obtenção de mudas.

Os resultados obtidos nestes experiementos evidenciam que as estacas de raízes de Brosimum gaudichaudii, mesmo sem aplicação de reguladores de crescimento apresentaram boa capacidade de enraizamento e pegamento (Figura 1) podendo ser utilizadas para a propagação vegetativa desta espécie, utilizando como substrato a areia, preferencialmente, acrescida de $25 \%$ de substrato comercial que

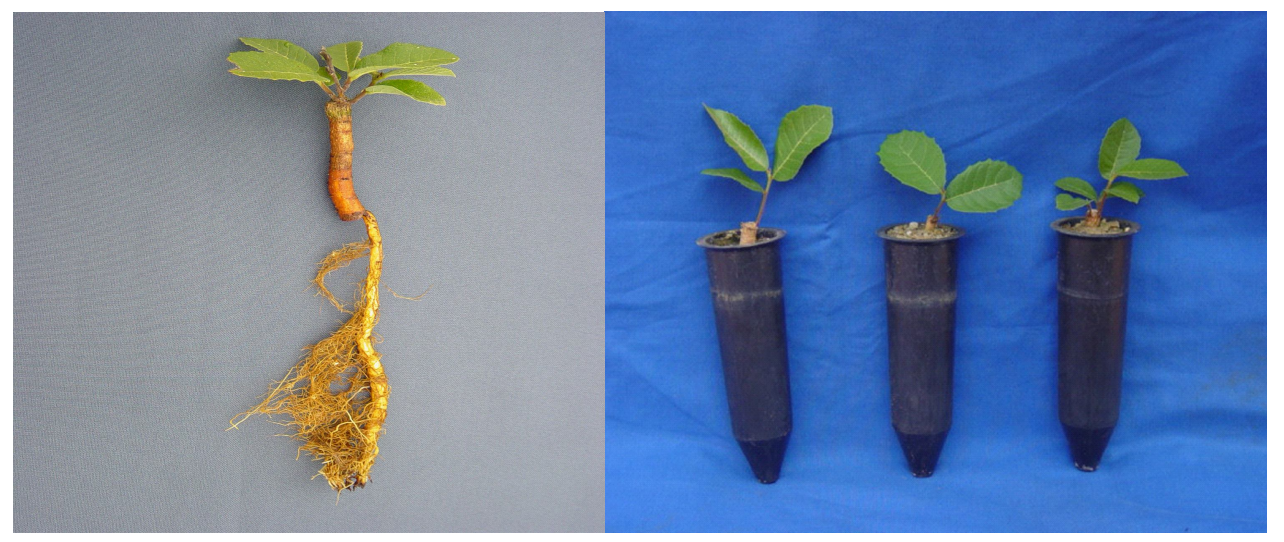

FIGURA 1. Mudas de Brosimum gaudichaudii Tréc. produzidas a partir de estacas de raiz em tubetes.

Rev. Bras. PI. Med., Botucatu, v.13, n.2, p.151-156, 2011. 


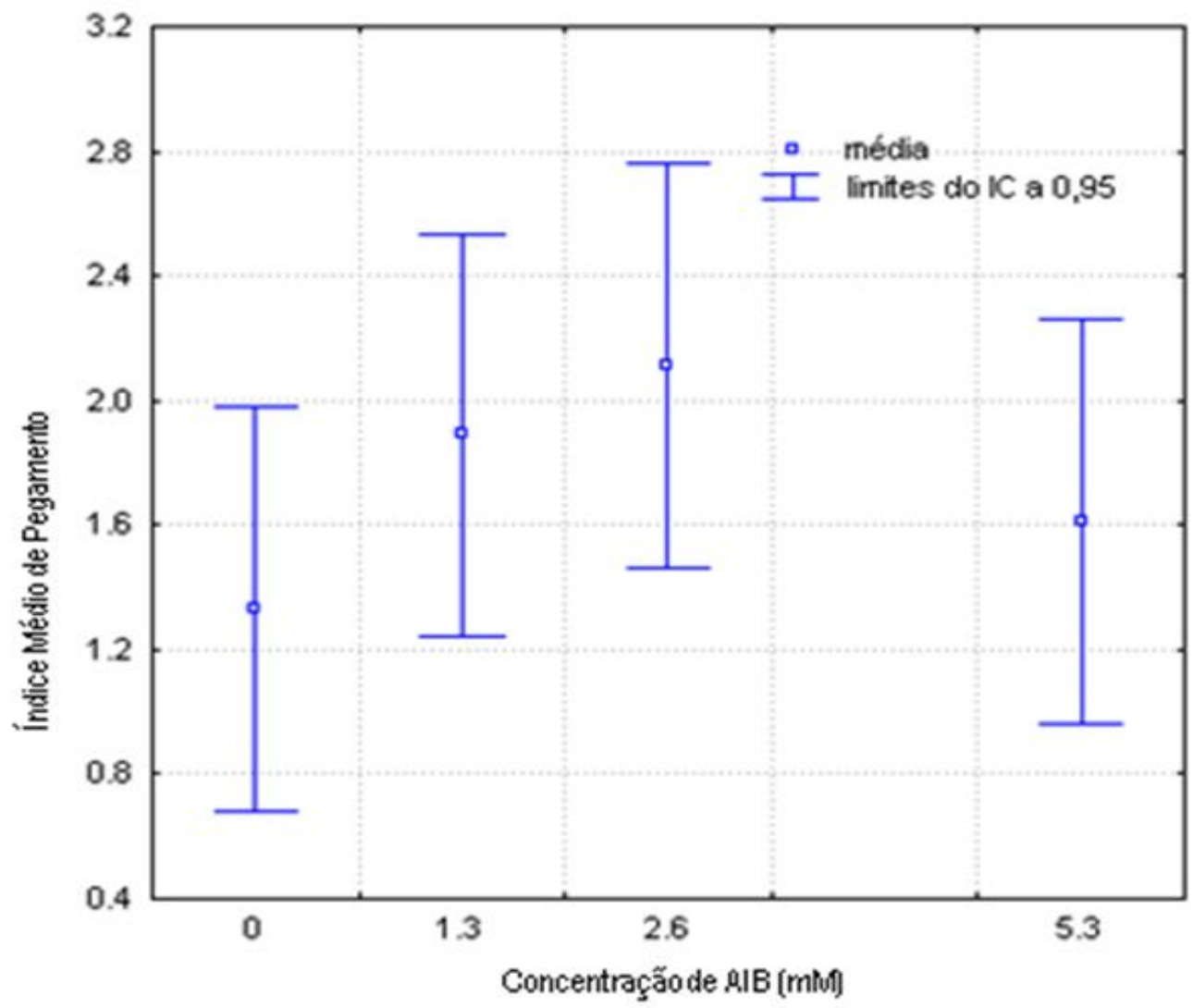

FIGURA 2. Efeito de doses de AIB sobre o Índice médio de pegamento.

TABELA 2. Efeito das doses de ANA sobre a Altura da maior brotação (AMB), Número médio de folhas das brotações (NF), Número médio de brotos (NB) e Índice médio de pegamento (IMP)*.

\begin{tabular}{lcccc}
\hline Doses $(\mathrm{mM})$ & AMB $(\mathrm{cm})$ & NF & NB & IMP \\
\hline 0 & 2,38 & 2,32 & 1,02 & 1,33 \\
1,229 & 2,37 & 1,64 & 1,14 & 1,00 \\
2,459 & 1,90 & 2,46 & 1,42 & 1,27 \\
4,918 & 1,38 & 1,38 & 0,91 & 1,16 \\
\hline C. V. (\%) & 78,95 & 67,95 & 44,67 & 35,72 \\
\hline
\end{tabular}

* As médias não apresentaram diferença significativa entre si ao nível de $5 \%$ de probabilidade pelo teste de Tukey.

TABELA 3. Efeito das doses de AIB sobre a altura da maior brotação (AMB), número médio de folhas das brotações (NF) e Número médio de brotos (NB)*.

\begin{tabular}{lccc}
\hline Doses $(\mathrm{mM})$ & AMB $(\mathrm{cm})$ & $\mathrm{NF}$ & $\mathrm{NB}$ \\
\hline 0 & 2,38 & 2,32 & 0,97 \\
1,3426 & 2,77 & 3,33 & 1,45 \\
2,6852 & 2,36 & 2,46 & 1,18 \\
5,3706 & 3,31 & 3,64 & 1,14 \\
\hline C. V. $(\%)$ & 78,95 & 67,95 & 44,67 \\
\hline
\end{tabular}

* As médias não apresentaram diferença significativa entre si ao nível de 5\% de probabilidade pelo teste de Tukey. 
favoreceu a uma melhor agregação física das raízes. Esta técnica poderá ser utilizada como estratégia para a conservação de recursos genéticos e para a produção de mudas clonais.

\section{REFERÊNCIA}

ALMEIDA, S.P. et al. Cerrado: espécies vegetais úteis. Planaltina: EMBRAPA-CPAC, 1998. 464p.

ANATEL, R.L. et al. Avaliação clínica toxicólogica preliminar do Viticromin ${ }^{\circledR}$ em pacientes com vitiligo. Revista Eletrônica de Farmácia, v.2, n.1, p.15-23, 2005. BENNET, J.C.; PLUM, F. Cecil - tratado de medicina interna. 20.ed. Guanabara: Koogan. 1996. v.2, 2647p. BUCHER, J.P. Aspéctos de conservação in vitro e micropropagação de mama-cadela (Brosimum gaudichaudii Tréc., Moraceae). 2002. 64p. Dissertação (Mestrado em Botânica) - Departamento de Botânica, Universidade de Brasília, Brasília.

CORRÊA, R.S.; MELO FILHO, B.; BAPTISTA, G.M.M. Avaliação fitossociológica da sucessão autogênica em áreas Mineradas no distrito federal. Cerne, v.13, n.4, p.406-15, 2007.

ELLIS, R.H.; HONG, T.D.; ROBERTS, E.H. An intermediate category of seed storage behavior? I. Coffee. Journal of Experimental of Botany, v.41, p.1167-74, 1990.

ELLIS, R.H. Seed storage behavior in Elaeis guinaensis. Seed Science Research, v.1, p.99-104, 1991.

FACHINELLO, J.C. Propagação vegetativa por estaquia. In: FACHINELLO, J.C.; HOFFMANN, A.; NACHTIGAL, J.C. Propagação de plantas frutíferas. Brasília: Embrapa Informação Tecnológica, 2005. p.13-43.

FACHINELLO, J.C. et al. Propagação de plantas frutíferas de clima temperado. 2.ed. Pelotas: Universitária, 1995. 178p.

FIDELIS, I. Micropropagação de mamacadela (Brosimum gaudichaudii), uma espécie medicinal. 1998. 109p. Dissertação (Mestrado em Agronomia) Universidade Federal de Lavras, Lavras.

HARTMANN, H.T. Plant propagation: principles and practices. 7.ed. New Jersey: Prentice Hall, 2002. 880p. HINOJOSA, G.F. Auxinas em plantas superiores: síntese e propriedades fisiológicas. In: BARRUETO, L.P.C. Hormônios vegetais em plantas superiores. Brasília: Embrapa Recursos Genéticos e Biotecnologia, 2005. p.15-57.

HOFFMANN, A.; NACHTIGAL, J.C.; FACHINELLO, J.C. Infra-estrutura para propagação de plantas frutíferas. In: FACHINELLO, J.C.; HOFFMANN, A.; NACHTIGAL, J.C. Propagação de plantas frutíferas. Brasília: Embrapa Informação Tecnológica, 2005. p.155-73.

JACOMASSI, E.; MOSCHETA, I.S.; MACHADO, S.R. Morfoanatomia e histoquímica de Brosimum gaudichaudiiTréc. (Moraceae). Acta Botânica Brasileira, v.21, n.3, p.575-97, 2007.

LORENZI, H. Árvores Brasileiras: manual de identificação e cultivo de plantas arbóreas nativas do Brasil. 2.ed. Nova Odessa: Editora Plantarum, 1988. 368p.

MACHADO, J.; PARENTE, T. Germinação de seis espécies frutíferas nativas do cerrado em condições de campo. Revista Brasileira de Fruticultura, v.8, n.1, p.358, 1986.

MACHADO, J.W.B.; PARENTE, T.V.; LIMA, R.M. Informações sobre germinação e características físicas das sementes de fruteiras nativas do Distrito Federal. Revista Brasileira de Fruticultura, v.8, n.2, p.59-62, 1986. MARTINS, M.V.M. Micropropagação de mama-cadela (Brosimum gaudichaudii Tréc.) uma espécie medicinal do cerrado. 1998. 50p. Dissertação (Mestrado - Área de Concentração em Agronomia) - Universidade de Brasília, Brasília.

MELO, J.T. Coleta, propagação e desenvolvimento inicial de espécies do cerrado. In: ALMEIDA, S.P. Cerrado: ambiente e flora. Planaltina: EMBRAPA-CPAC, 1998. p.195-243.

PALHARES, D.; DE PAULA, J.E.; SILVEIRA, C.E. Morphology of stem and subterranean system of Brosimum gaudichaudii Tréc. (Moraceae). Acta Botannica Hungarica, v.48, n.1-2, p.89-101, 2006.

PENHA, A.S. Influência da propagação vegetativa a partir de raízes gemíferas nas características populacionais de espécies arbóreas em fragmentos de florestas tacionais semideciduais. 2004. 135p. Dissertação (Doutorado em Biologia Vegetal) - Universidade Estadual de Campinas, Campinas.

$\mathrm{PIO}, \mathrm{R}$. Enraizamento de estacas radiculares de figueira 'roxo de Valinhos' tratadas com AIB e dois métodos de imersão. Scientia Agraria, v.9, n.1, p.111-5, 2008.

POZET, G.L. Brosimum gaudichaudii Trec. (Moraceae): da planta ao medicamento. Revista de Ciências Farmacêuticas Básicas e Aplicada, v.26, n.3, p.159-66, 2005.

POZET, G.L.; BERNARDI, A.C. Contribuição ao estudo químico de Brosimum gaudichaudii Tréc. Revista da Faculdade de Farmácia e Odontologia de Araraquara, v.3, p.215-23, 1969.

RIZZINI, C.T.; HERINGER, E.P. Estudo sobre sistemas subteraneos difusos de plantas campestres. Anais da Academia Brasileira de Ciências, v.38, p.85-112, 1966. ROBERTS, E.H. Predicting the storage life of seeds. Seed Science and Technology, v.1, p.499-514, 1973.

SALES, D. Germinação de sementes de Brosimum gaudichaudii Trécul submetidas a diferentes prétratamentos. Acta Horticulturae, v.569, p.137-40, 2002. SILVA, D.B. et al. Frutas do Cerrado. Brasília: Embrapa Informação Tecnológica, 2001. 178p.

SILVA, D.B. et al. Conservação de germoplasma semente em longo prazo. In: NASS, L.L. Recursos genéticos vegetais. Brasília: Embrapa Recursos Genéticos e Biotecnologia, 2007. p.441-71.

THOMAS, C.L. (Coord.) Dicionário médico enciclopédico Taber. 17.ed. São Paulo: Manole, 2000. 2227p.

VIEIRA, R.F. Estratégias para conservação e manejo de recursos genéticos de plantas medicinais e aromáticas. Brasília: Embrapa Recursos Genéticos e Biotecnologia, Instituto Brasileiro do Meio Ambiente e dos Recursos Naturais Renováveis (Ibama), Conselho Nacional de Desenvolvimento Científico e Tecnológico CNPq, 2002. 59p.

WETZEL, M.M.V.S. Época de dispersão e fisiologia de sementes do cerrado. 1997. 168p. Dissertação (Doutorado em Ecologia) - Universidade de Brasília, Brasília. 